\title{
Continuous Target Tracking Algorithm in Aerial Wireless Sensor Network
}

\author{
Yaoyao Jiao, Li Tan*, Weijun Su and Minghua Yang \\ School of Computer and Information Engineering, Beijing Technology and Business University, Beijing 100048, China \\ *Corresponding author
}

\begin{abstract}
In three-dimensional space, the traditional algorithm of virtual force applies to continuous target tracking process is prone to problems of distraction and shorter time in keeping tracking of the target. Aiming at solving these problems, this paper proposed a suitable three-dimensional space deployment algorithm apply to continuous target tracking. The virtual force in this algorithm is combined with the inter-node force, obstacle repulsion, attractions generated by monitored-path and tracking target. It can make the nodes cover the target paths even and sustained. Performance of simulations show that, this algorithm obtains obvious improvement in continuous target tracking compared to the traditional virtual force.
\end{abstract}

Keywords-continuous target tracking; aerial wireless sensor network; combined virtual force

\section{RELATED WORKS}

Deployment tactics is one of the most key issues in the target tracking, because it directly impacts on the tracking effect, and then further determines the integrity, accuracy and real-time performance of data collection. As in AWSN, nodes' deployment is executed in three-dimensional space that developed from the original two-dimensional space, and the deployment region varies from two-dimensional to threedimensional[1-4]. Nowadays, many two-dimensional deployment algorithms are no longer applicable. Therefore, new methods and ideas are urgently needed in design of deployment algorithm[4-5].

In target tracking, the most concern is no longer the coverage quality of the entire monitoring area, but the path that a moving target passed by when the target is caught, i.e. the core issue of coverage is the probability monitored as soon as when a moving target along any path passing through the nodes deployment area. Zhang et al. put forward the DCTC (Dynamic convoy tree-based collaboration)[6] which organizes sensor nodes nearby to form a small dynamic cluster based on the actual location of the target, then makes a locating and forecast. When the target transfers to another location, the algorithm will dynamically increases or deletes sensor nodes to build up a new dynamic cluster. Reference[7] proposed an algorithm of target tracking on static clusters by setting fit network topology to reduce the energy consumption and to obtain higher amount of information. However, although the algorithm is capable to save energy, the possibility of tracking failure rate is relatively higher, so that will course reducing of the reliability of tracking.

Aimed at the issue of monitoring on target tracking phase, Tao Dan et al. [8] proposed a path coverage enhancement algorithm based on virtual field that turns the matter of coverage enhancement into virtual force between inter-nodes and target-nodes by introducing the concept of "node central point" and "target trajectory point". The algorithm can reach the maximization of the path coverage.

However, the algorithms mentioned above are mostly applied in two-dimensional space, and the tracking nodes are static. It is the process of waking the "right" dormant nodes adjacent to the target path to join the target tracking by collaboration of multiple sensor nodes. Although some algorithms added a directional function to sensor nodes, which helps improving the control of target tracking, enriched the study content, yet they are still not adequate for continuous target tracking, especially in circumstances of battle. 1-2 decoys usually released shielding for the real one behind. It will attract much attention resulting in the deployment chaos and coverage blind zones. Effective tracking can no longer be kept on when the subsequent real targets appear.

To solve these problems, this article proposes a continuous target tracking algorithm in AWSN(Aerial Wireless Sensor Network) which achieves a sustained and even coverage on monitoring path, as well as gains an effective tracking of continuous targets appear around the monitor path .

\section{TARget Tracking Algorithm Model}

\section{A. Assumptions and Definitions}

In the first place put forward the following hypothesis and definitions:

Hypothesis 1 Each node could calculate its own position by the algorithm, and obtain the determined coordinates information.

Hypothesis 2 Take one target as a node. The same are true as nodes, the center, i.e. the central coordinates is taken as its estimated position.

Hypothesis $3 R c$ means the communication radius of node, node $i$ and $j$ become neighbor nodes which can communicate with each other when their rectilinear distance dij is less than or equal to $R c$.

Hypothesis 4 Communication energy is continuous within $R c$ while out of $R c$ is none.

Definition 1 Node model is [O $(x, y, z), R m, R k, R c, F \max ]$. Where $O(x, y, z)$ is the three-dimensional coordinate values of 
nodes, $x, y, z$ respectively represents coordinate values on the $\mathrm{X}$ axis, $\mathrm{Y}$ axis, $\mathrm{Z}$ axis in rectangular coordinate system; $R m$ is the minimum radius value between two nodes occur collision, shortened as the minimum radius; $R k$ is equilibrium radius. When the straight-line distance between two nodes equals to $R k$, no force acts on nodes; $R c$ is the communication radius; Fmax is the maximum repulsion when the distance between nodes less than $R m$.

Definition 2 The curve model of monitoring path is $[f(x, y, z) \operatorname{Lim} D P(x, y, z)]$, among which, $f(x, y, z)$ is the general equation of the curve, Lim represents the curve's set of maximum and minimum values on $x, y, z-$ axis coordinates in the region of space, that are used to limit the largest deployment area of all nodes. The shortest distance of the set of all distances from any node to the target curve is defined as $D$, whereas the corresponding coordinates on the target path is named as $P$.

\section{B. Combination Virtual Force Model}

Node is affected by four types of forces in aerial wireless sensor network.

\section{1) Inter-Node Force}

Based on swarm intelligence concept, the movable nodes in aerial wireless sensor network can be considered as charged particles that the nodes are assumed to be charged particles must have attraction and repulsion in physics point of view. So there is an electric field in which the nodes are affected by the electric forces that depends on the distance between two nodes. When it turns out to be attraction, it indicates that the distance between two nodes is too far away. On the contrary, they are too much close.

\section{2) Obstacle Force}

Practically, there definitely being obstacles in surroundings, the nodes are affected by the forces that generated by obstacles. In order to avoid collisions leading to equipment damage or network paralysis, assuming repulsion is produced to nodes by the obstacles in the target area.

\section{3) Monitored-Path Force}

Suppose the nodes are given a force generated by monitored-path. In order to guarantee there's no coverage hole caused on monitored- path due to the nodes tracking targets, turn the path into a curve with attraction.

\section{4) Tracking Target Force}

Nodes are bound to be affected by an attraction force that is generated by the tracked target. While the nodes monitored a movable target within its sensible scope, they shall be tightly tracking the monitored target due to such attraction force and complete the tracking task with the coordination of various forces.

It commonly uses vector to represent the forces by which the nodes are affected and the vector superposition builds up the resultant force. The nodes in aerial wireless sensor network adjust their positions timely under the resultant force. When a node is affected by no forces or reach the upper limit of moving distance, it will stop moving. Nodes can achieve self- deployment and complete the accurate tracking of moving targets under the four types of force action.

- Inter-node force

Taken the set of nodes in AWSN as a virtual physical system containing a force $F$, mass $\mathrm{m}$ and other parameters, there is existing either attraction or repulsion between each node, the node $i$ is affected by a force from its neighbor node $j$. Moving direction and distance of node $i$ depend on its location in the network, its mass $m i$, the resultant force $F i$ contains attraction and repulsion it affected from its neighbor nodes and other parameters. Within the range of communication distance $R c$, there is an interactive force between nodes, turning out to be attraction or repulsion whose degree links to the distance between nodes, meaning the nature of force is determined by the distance.

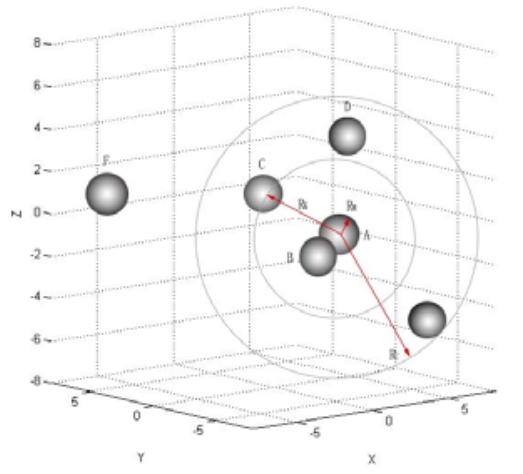

FIGURE I. RELATION OF INTER-NODE FORCE

As shown in Fig. 1, there are 6 nodes from node $A$ to $F$ in three-dimensional space, out of which node A's minimal radius is $R m$, equilibrium radius is $R k$ and communication radius is $R c$.

The analysis of distance and force between any two nodes is shown as follows:

a) In Fig. 1, When the distance between node $\mathrm{A}$ and $\mathrm{B}$ meets $d A B=R m$ (minimum radius), these two nodes come into a collision. Meantime the inter-node force is up to maximum Fmax;

b) In Fig. 1, When the distance between node $\mathrm{A}$ and $\mathrm{C}$ meets $R m<d A C<R k$ (equilibrium radius), the inter-node force shows as repulsion $F \tau$ which decreases whilst the rectilinear distance $d A C$ between them increases;

c) In Fig. 1, At the time when the distance between node A and $\mathrm{D}$ meets $d A D=R k$, attraction and repulsion between them are equal, meaning the resultant force is zero;

d) In Fig. 1, the distance between node A and D meets $R k<d A C<R c$ (communication radius), the inter-node force shows as repulsion $F \alpha$ which decreases as the rectilinear distance $d A E$ between them increases;

e) In Fig. 1, While the distance between node A and F meets $\mathrm{dAF}>\mathrm{Rc}$, the active force between them is zero.

The force $F \alpha$ and $F \tau$ between two nodes can be represented as formulas (1) and (2) as the following: 


$$
\begin{gathered}
F_{\alpha}(i, j)=\left\{\begin{array}{cc}
-\frac{k_{1} m_{i} m_{j}}{d(i, j)^{\alpha 1}} & 0<d_{i j}<R c \\
0 & d_{i j} \geq R c
\end{array}\right. \\
F_{\tau}(i, j)=\left\{\begin{array}{cc}
-\frac{k_{2} m_{i} m_{j}}{d(i, j)^{\alpha 2}} & 0<d_{i j}<R k \\
0 & d_{i j} \geq R k
\end{array}\right.
\end{gathered}
$$

In the above shown equations, $k 1, k 2, \alpha 1, \alpha 2$ are the gain coefficients; $m i$ and $m j$ are the mass factors of node, generally taken per unit $1 ; d(i, j)$ is the distance between node $i$ and $j, R c$ is the communication radius, and $R k$ is the equilibrium radius.

\section{- Obstacle force}

As mentioned above, nodes in AWSN are not only affected by inter-node force, but also obstacle force in actual environment being monitored. It is inevitable for nodes, when they are in movement of deployment to meet unpredictable obstructions impacting their moving. Nodes collided with an obstacle will cause unnecessary losses, therefore, to define the obstacle force as repulsion. Setting obstacles put repulsion on all nodes, and the value of force is also determined by the distance between the node and obstacle as shown in Fig. 2.

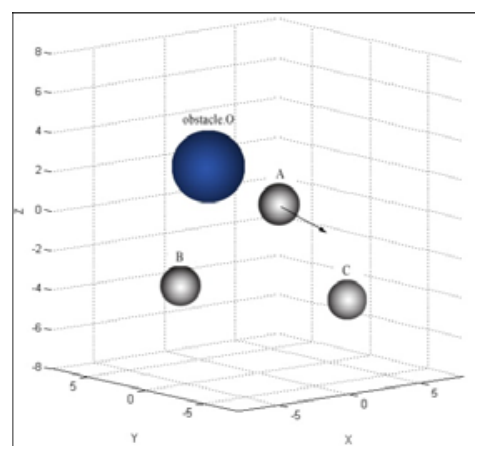

FIGURE II. OBSTACLE FORCE

Obstacle $\mathrm{O}$ has its own range of repulsion field. When the node A moves into this range, it will be affected by the repulsion pointed from obstacle $\mathrm{O}$ to node $\mathrm{A}$. The force can be calculated as formulas (3) below:

$$
F_{o}(i, o)=\left\{\begin{array}{cl}
-\frac{k_{3} m_{i} m_{o}}{d(i, o)^{\alpha 3}} & 0<d_{i o}<L \\
0 & d_{i o} \geq L
\end{array}\right.
$$

In this formula, $k 3$ and $\alpha 3$ are the gain coefficients; $m i$ and $m o$ are the mass factors of node, generally taken per unit length; $d(i, o)$ is the distance between node $i$ and obstacle; $L$ is the radius of repulsion field, i.e. the effective distance of node effected by the obstacle repulsion.

- Monitored-path force
In order to ensure the nodes on monitored-path are not led to coverage holes due to the tracking targets, set the monitoredpath could generate attraction to each node, under which the nodes will autonomously move to the path so that the absence of coverage is avoided, and every mobile target can be tracked effectively and accurately ensured.

For any node, named A, it is sure to find the shortest path to arrive the monitored-path, which is named D whose corresponding spot on monitored-path is defined as projected point $\mathrm{P}$ of the node on the curve. Now conduct limited point segment on the curve so as to explore the shortest segment point to node $\mathrm{A}$ and nominated which as projection point $\mathrm{P}$. Then there exists attraction between $\mathrm{P}$ and $\mathrm{A}$, and it is pointed from $\mathrm{P}$ to $\mathrm{A}$. The attraction generated by projection point $\mathrm{P}$ can make nodes move toward monitored-path always along the "shortest path", achieving full coverage as shown in Fig. 3.

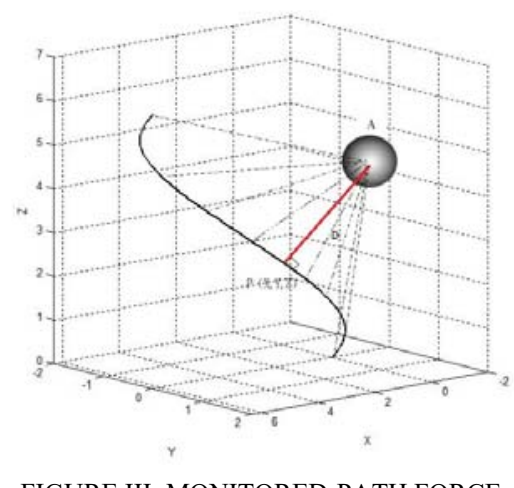

FIGURE III. MONITORED-PATH FORCE

The force can be calculated by formulas (4) as follows:

$$
F_{p}(i, P)=\left\{\begin{array}{c}
-\frac{k_{4} m_{i} m_{p}}{d(i, P)^{\alpha 4}} \quad d_{i P} \geq d \\
0 \quad d_{i P}<d
\end{array}\right.
$$

In this equation, $k 4$ and $\alpha 4$ are gain coefficients; $m i$ and $m p$ are mass factors of node, generally taken per unit length; $d(i, P)$ is the distance between nodes and the perpendicular foot. $d$ is the maximum distance beyond which nodes can't be attracted by the monitored-path.

- Tracking target force

In order to achieve the tracking and monitoring over the moving targets, set a much stronger repulsion force between the tracked target and the nodes than that of the inter-nodes. When the tracked targets appear, nodes can be quickly attracted in good time within its communication range as shown in Fig. 4. 


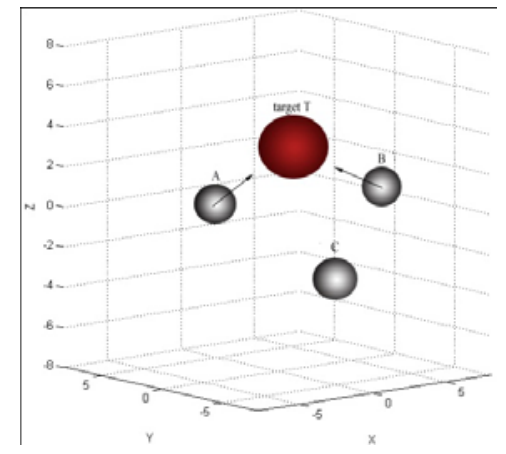

FIGURE IV. TRACKING TARGET FORCE

The attraction generated by target $\mathrm{T}$ can be calculated with the following formulas (5):

$$
F_{T}(i, T)=\left\{\begin{array}{c}
-\frac{k_{5} m_{i} m_{T}}{d(i, T)^{\alpha 5}} \quad d_{i T}<r \\
0 \quad d_{i T} \geq r
\end{array}\right.
$$

In this formula equation, $k 5$ and $\alpha 5$ are gain coefficients; $m i$ and $m T$ are mass factors of nodes, generally taken per unit length; $d(i, T)$ is the distance between the nodes and the target. $r$ is the maximum distance beyond which nodes can't be attracted by the target.

\section{PERFORMANCE ANALYSIS}

In the experiments, the traditional algorithm and improved algorithms were applied in the experiment for continuous target tracking. Contrast performances are shown in Fig. 5. When there is only one target, the results of both algorithms are good, but the traditional algorithm is significantly inferior to the improved one with the increase of targets. Because a good number of the deployed nodes have been "taken away" when the first moving target appeared, that destroyed the stable state of the original monitoring path deployment. Coverage holes appeared surrounding the path by solely relying on inter-node force. When the next target made a show, the tracking could not be carried out timely and accurately owing to no nodes coverage toward the monitored-path timely supplemented, and the time duration of effective tracking was shortened as well. On the contrary, since there existing attraction of the monitored-path by the improved algorithm, the time of continuous target tracking could maintain at a relatively stable state. It reasons why is the nodes were not only subjected to inter-node force, but also to the attraction of the monitored-path. At a time when the nodes near the path were tracking on the moving target and left the original deployed position, those nodes were far away from the path toward which the nodes would have been getting closer under the resultant force, hence the timely supplement was thus accomplished, so that the tracking task could still have been completed when the next moving target emerged, and so such circulation is kept on (to guarantee the sufficiency of nodes deployment).

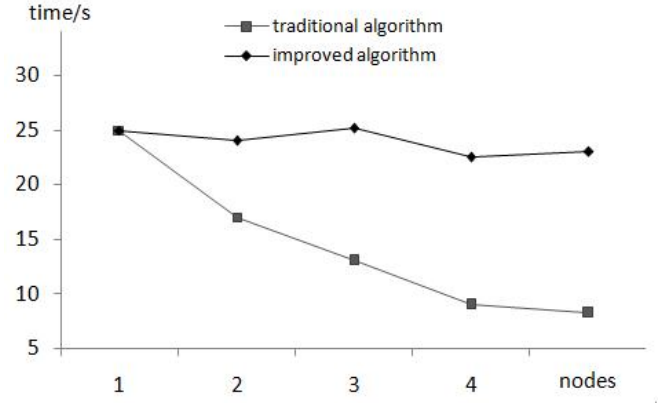

FIGURE V. DURATION OF CONTINUOUS TARGET TRACKING

\section{CONCLUSION}

This paper presents an algorithm applicable to the continuous targets tracking. Its main contribution is able to resolve the problem of timely supplement of the missing nodes during the process of continuous tracking on targets, meeting the demands in actual environment operation, materialized the self- deployment of nodes and tracking on continuous moving targets in a three-dimensional space. The simulation experiments' result demonstrated the effectiveness of the algorithm.

\section{ACKNOWLEDGMENT}

We are grateful for the supported by Beijing Natural Science Foundation (4132025), Beijing Higher Education Young Elite Teacher Project (YETP1448).

\section{REFERENCES}

[1] K. Dantu, M. Rahimi, H. Shah, S. Babel, A.Dhariwal, and G.S.Sukhatme.Robomote. enabling mobility in sensor networks.In Proceedings of the 4th international symposium on Information processing in sensor networks, IEEE Press, 2005:55

[2] Vasilescu I, Kotay K, Rus D, et al. Krill: an exploration in underwater sensor networks[C]// Embedded Networked Sensors, 2005. EmNetS-II. The Second IEEE Workshop on. IEEE, 2005:151-152.

[3] Zhang M J, Cui J, University L T. Prediction Model of Moving Target Tracking in Wireless Sensor Networks[J]. Computer Systems \& Applications, 2014, 3(3):258-271.

[4] Choi I D, Park J S, Lee K H, et al. The three dimensional node deployment for Sensor Network[C]//Advanced Software Engineering and Its Applications, 2008. ASEA 2008.IEEE, 2008: 271-275.

[5] Placzek B. Communication-aware algorithms for target tracking in wireless sensor networks[J]. Communications in Computer \& Information Science, 2014:69-78.

[6] Zhang W, Cao G. DCTC: dynamic convoy tree-based collaboration for target tracking in sensor networks[J]. IEEE Transactions on Wireless Communications, 2004, 3(5):1689-1701.

[7] Tu Z L, Wang Q, Shen Y. A Distributed Simultaneous Optimization Algorithm for Tracking and Monitoring of Moving Target in Mobile Sensor Networks[J]. Acta Automatica Sinica, 2012, (3):452-461.

[8] Tao D, Research on Coverage Control and CooPerative Processing Method for Video Sensor Networks[D]. Beijing,Beijing University of Posts and Telecommunications,2007:67-70. 